\title{
Renal Parenchyma Thickness: A Rapid Estimation of Renal Function on Computed Tomography
}

\author{
Daniel M. Kaplon, Michael S. Lasser, Mark Sigman, George E. Haleblian, Gyan Pareek
}

Section of Minimally Invasive Urologic Surgery, Department of Surgery, Division of Urology, The Warren Alpert School of Medicine of Brown University, Providence, Rhode Island, USA

\begin{abstract}
Purpose: To define the relationship between renal parenchyma thickness (RPT) on computed tomography and renal function on nuclear renography in chronically obstructed renal units (ORUs) and to define a minimal thickness ratio associated with adequate function.

Materials and Methods: Twenty-eight consecutive patients undergoing both nuclear renography and CT during a six-month period between 2004 and 2006 were included. All patients that had a diagnosis of unilateral obstruction were included for analysis. RPT was measured in the following manner: The parenchyma thickness at three discrete levels of each kidney was measured using calipers on a CT workstation. The mean of these three measurements was defined as RPT. The renal parenchyma thickness ratio of the ORUs and non-obstructed renal unit (NORUs) was calculated and this was compared to the observed function on Mag-3 lasix Renogram.

Results: A total of 28 patients were evaluated. Mean parenchyma thickness was $1.82 \mathrm{~cm}$ and $2.25 \mathrm{~cm}$ in the ORUs and NORUs, respectively. The mean relative renal function of ORUs was 39\%. Linear regression analysis comparing renogram function to RPT ratio revealed a correlation coefficient of $0.48(\mathrm{p}<0.001)$. The linear regression equation was computed as Renal Function $=0.48+0.80 *$ RPT ratio. A thickness ratio of 0.68 correlated with $20 \%$ renal function.

Conclusion: RPT on computed tomography appears to be a powerful predictor of relative renal function in ORUs. Assessment of RPT is a useful and readily available clinical tool for surgical decision making (renal salvage therapy versus nephrectomy) in patients with ORUs.
\end{abstract}

Key words: kidney; obstruction; kidney cortex; computed tomography

Int Braz J Urol. 2009; 35: 3-8

\section{INTRODUCTION}

The most comprehensive radiographic modality for renal imaging is computed tomography (CT). As CT technology has advanced with the development of helical scanners, the resulting superior spatial and temporal resolution no longer limits the role of CT to assess static anatomic parameters (1). In recent years, multiple studies have demonstrated a clear role for utilizing $\mathrm{CT}$ to assess renal perfusion and glomerular filtration rate (GFR) (2,3). Langheinrich et al. dem- onstrated that GFR can be measured accurately with triphasic CT (4). In addition, Patlak et al. developed a model for determining tissue function based on contrast transfer between tissues; this technique has been extrapolated to renal function in several studies (5). The information provided by these functional studies may be useful in defining recoverability of renal function, an important concept in assessing the viability of an obstructed renal unit (ORU). Other investigators have also studied anatomical details for assessing renal function with a recent report analyzing the role 
of renal parenchyma thickness (RPT) on ultrasound as a predictor of recoverability of renal function (6).

We sought to define the relationship between RPT on computed tomography and renal function on Mag-3 Lasix renogram in chronically obstructed renal units (ORUs) in order to define a minimal thickness ratio associated with adequate function. Our goal was to provide an efficient and pragmatic clinical tool for surgical decision making in patients with chronic ORUs.

\section{MATERIALS AND METHODS}

Institutional review board approval was obtained prior to the commencement of the study. Fifty two consecutive patients undergoing lasix renograms between 2004 to 2006 were reviewed. From this cohort those who had a concomitant CT scan within 6 months were extracted. Patients with bilateral hydronephrosis, solitary kidney, and medical renal disease were excluded from review. In addition, those patients whose history suggested acute obstruction were excluded as well. Of this cohort, 28 patients had a diagnosis of unilateral obstruction and were included for analysis. Mean and median age was 49 and 48 , respectively.
A General Electric Hi-Speed Advantage CT scanner was used to evaluate all patients in the study. RPT was measured at a hilar image on CT scan of the ORU and compared to a corresponding image of the NORU (Figure-1). The parenchyma thickness at the exact cranio-caudal midpoint of each kidney was measured using calipers on a CT workstation. The measurement was taken at an angle exactly perpendicular to the axis of the kidney. All measurements were performed by a single investigator. Two additional measurements were obtained in each kidney: one 2 $\mathrm{cm}$ cranial to the midpoint and one $2 \mathrm{~cm}$ caudal to the midpoint. For all scans, the parenchyma was measured from the renal capsule to the edge of the collecting system. The mean of the three measurements was then taken for each kidney and defined as the RPT. The renal parenchyma thickness ratio of the ORUs and non-obstructed renal unit (NORUs) was calculated and this was compared to the observed function on Mag-3 lasix Renogram. Regression analysis was performed using WESSA statistical software (Wessa, P. (2009), Free Statistics Software, Office for Research Development and Education, version 1.1.23-r3, URL http://www.wessa.net/).

Correlation between RPT ratio and function was determined. Further analysis of the linear regression curve allowed for calculation of the RPT ratio

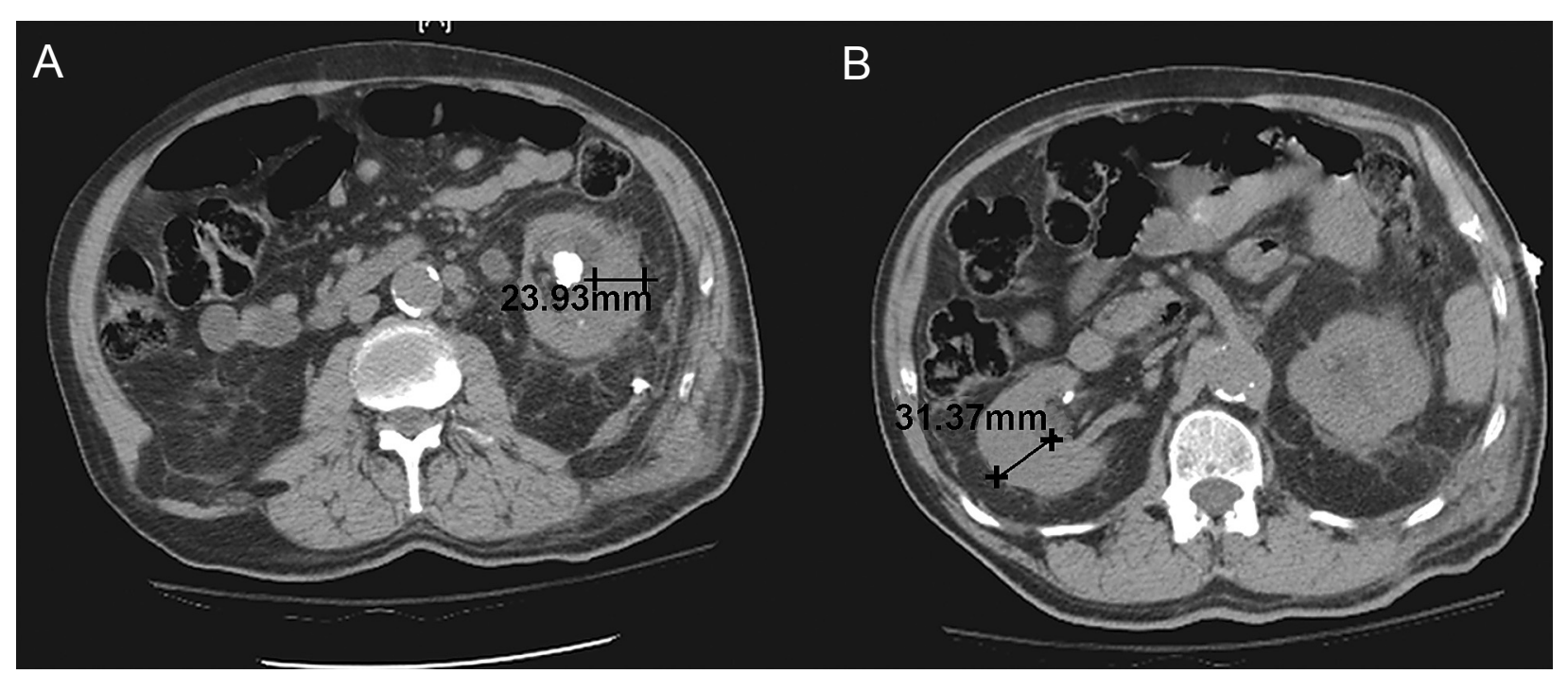

Figure 1 - Measurement of renal parenchyma thickness for obstructed renal unit (A) and non-obstructed renal unit (B). 
correlating to a renal function of $20 \%$, which at our institution represents the general cutoff for performing renal salvage surgery.

\section{RESULTS}

A total of 28 patients were evaluated. Mean patient age was 49 years (range 13-90 years). Obstruction was secondary to ureteropelvic junction obstruction $(\mathrm{n}=12)$, calculus disease $(\mathrm{n}=5)$, malignancy $(\mathrm{n}=2)$, stricture $(\mathrm{n}=1)$, ureterocele $(\mathrm{n}=1)$, and non-specified hydronephrosis $(n=6)$. Seventeen patients were evaluated with noncontrast $\mathrm{CT}$ scan and 10 patients with contrast $\mathrm{CT}$ scan.

Mean parenchyma thickness was $1.82 \mathrm{~cm}$ and $2.25 \mathrm{~cm}$ in ORUs and NORUs, respectively. The mean renal function of ORUs was $39 \%$ and the mean renal function of NORUs was $61 \%$.

Linear regression analysis comparing renogram function to ratio of thicknesses between the ORUs and NORUs revealed a correlation coefficient of $.48(p<0.001)$ (Figure-2). The linear regression equation was computed as Renal Function $=.0 .48$ $+0.80 * \mathrm{RPT}$ ratio $* \mathrm{RPT}$ ratio. A thickness ratio of 0.68 correlated with $20 \%$ renal function.

\section{COMMENTS}

Computed tomography has become a firstline assessment tool for patients presenting with renal obstruction and has proven to be the most effective modality for diagnosing the etiology of obstruction (7). Not only is CT widely used, it also provides a clear depiction of the renal anatomy and may provide additional valuable and quantifiable clinical information regarding renal function. An important parameter assessed on CT is the "health" of the renal parenchyma. Often in the outpatient setting RPT on CT scan may suggest diminished renal function. When this is observed, patients often undergo radionucleotide scans for a more formal evaluation of differential renal function. This information then helps the clinician and the patient make decisions about the appropriate course of treatment. Thus, our goals were to determine if a relationship between RPT on computed tomography and renal function on Mag-3 lasix renogram in ORUs exists and if so, to define a minimal thickness ratio associated with adequate function.

Recently, many studies have examined various parameters on CT in order to predict renal function. Evidence has shown the differential renal parenchyma volume measured by noncontrast $\mathrm{CT}$ provides

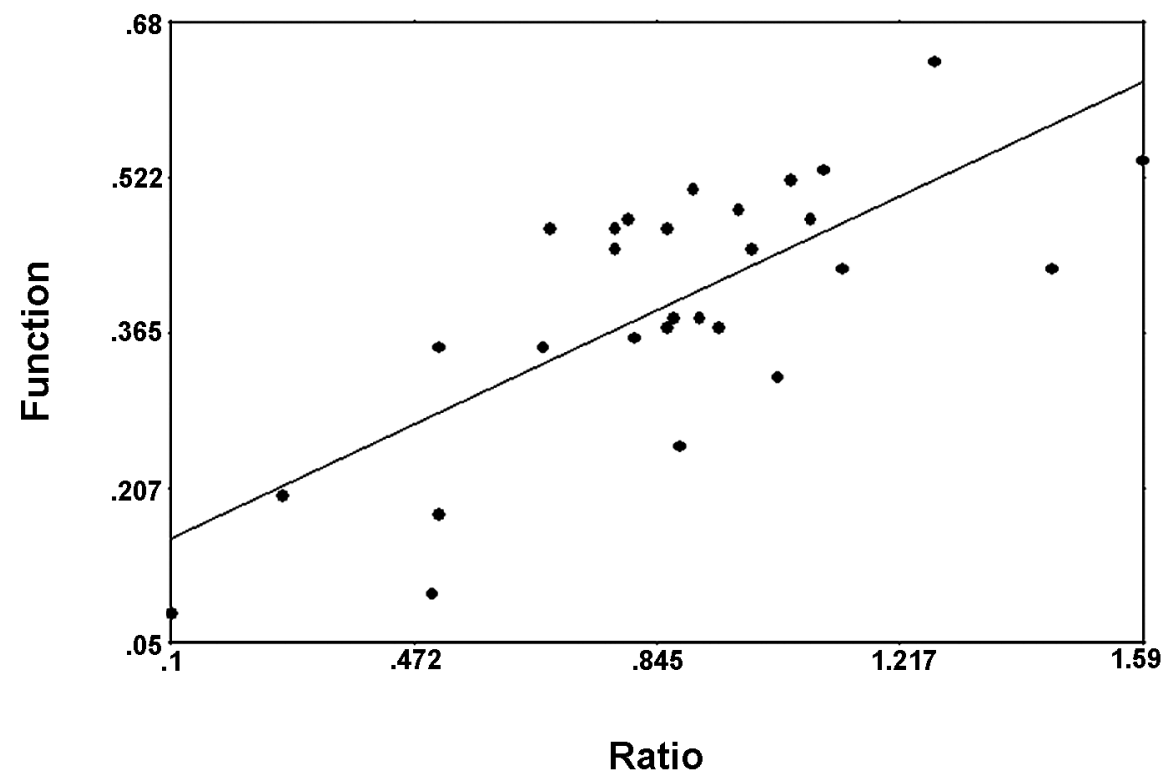

Figure 2 - Linear regression showing renal parenchyma thickness ratio vs. renogram function. 
a fairly accurate prediction of differential creatinine clearance (7). El-Dein et al. demonstrated that contrast enhanced spiral CT is as accurate as nuclear renography for calculating total and separate renal function (8). There are, however, major drawbacks to these forms of renal function estimation. Primarily, these studies required sophisticated software to calculate total renal volume based on CT reconstructions (8). Such scans must be performed as a dedicated study as data from existing scans may not be adequate for the sophisticated reconstructions and volume rendering required. In instances where reconstruction can be performed on existing scans, it is often a time consuming and costly endeavor. Measurement of RPT ratios, however, can be performed in a retrospective manner on existing scans, either with or without contrast.

Multiple recent studies have demonstrated the ability to calculate GFR using contrast enhanced CT. Most of these studies rely on a variation of the Patlak model, which involves using the transfer of contrast into tissue as a marker for function (5). While this method has been validated and provides an estimate of GFR, its practical use is limited by the complexity of calculation required to arrive at the GFR value. In addition, Hackstein has suggested that the Patlak model can overestimate GFR in several situations (3).

In patients with asymmetric renal disease such as renal artery stenosis and obstruction, measurement of unilateral function is not possible by calculation of global creatinine or inulin clearance because these parameters are influenced by the contralateral renal unit (1). Determination of individual kidney function has been historically performed either by percutaneous nephrostomy and creatinine clearance or by nuclear scintigraphy. Both of these modalities have limitations, with the former being invasive and the latter being often unreliable in cases of obstruction (9). More sophisticated models for predicting unilateral renal function include electron beam $\mathrm{CT}$, three-dimensional ultrasound, and gadolinium magnetic resonance (1).

The major limitation of this study is that the nuclear renogram is used as the gold-standard test for function. We chose the nuclear renogram because it is the most commonly used test for function of ORUs at most institutions, including our own. It is important to realize, however, that nuclear renogram can actually overestimate function in cases of obstruction (9). A more accurate assessment of renal function could be assessed via a percutaneous nephrostomy with subsequent creatinine clearance, but this was not possible due to the retrospective nature of this study. In addition, the population studied here is small. Admittedly, only 3 patients in our series had renal function less than $20 \%$ by renogram, so a larger population will be needed to further validate these results. In addition, it must be noted that patients with abnormalities of the measured area of the kidney such as focal scarring or fetal lobulation would not be good candidates for this method of measurement.

To date, no study has used parenchyma thickness alone to compare function to nuclear renography. We assessed a group of patients in whom all had a chronic unilateral abnormality and a presumed normal contralateral renal unit. This study describes an approach that allows for rapid estimation of renal function using a single measurement, which can be done without sophisticated reconstructions. The thickness ratio of the ORU to NORU correlates quite well with the relative function as predicted by nuclear renogram.

\section{CONCLUSION}

Renal parenchyma thickness ratio on computed tomography appears to be a powerful predictor of relative renal function in chronic ORUs. Assessment of RPT ratio is a useful clinical tool for surgical decision making in patients with ORUs. It relies on straight-forward measurements that can be made on existing CT scans without the need for complex calculations, reconstructions, or secondary studies.

\section{CONFLICT OF INTEREST}

None declared.

\section{REFERENCES}

1. Daghini E, Juillard L, Haas JA, Krier JD, Romero JC, Lerman LO: Comparison of mathematic models for 
assessment of glomerular filtration rate with electronbeam CT in pigs. Radiology. 2007; 242: 417-24.

2. O'Dell-Anderson KJ, Twardock R, Grimm JB, Grimm KA, Constable PD: Determination of glomerular filtration rate in dogs using contrast-enhanced computed tomography. Vet Radiol Ultrasound. 2006; 47: 12735.

3. Hackstein N, Bauer J, Hauck EW, Ludwig M, Krämer HJ, Rau WS: Measuring single-kidney glomerular filtration rate on single-detector helical CT using a twopoint Patlak plot technique in patients with increased interstitial space. AJR Am J Roentgenol. 2003; 181: 147-56.

4. Hackstein N, Wiegand C, Rau WS, Langheinrich AC: Glomerular filtration rate measured by using triphasic helical CT with a two-point Patlak plot technique. Radiology. 2004; 230: 221-6.

5. Patlak CS, Blasberg RG, Fenstermacher JD: Graphical evaluation of blood-to-brain transfer constants from multiple-time uptake data. J Cereb Blood Flow Metab. 1983; 3: 1-7.
6. Khalaf IM, Shokeir AA, El-Gyoushi FI, Amr HS, Amin MM: Recoverability of renal function after treatment of adult patients with unilateral obstructive uropathy and normal contralateral kidney: a prospective study. Urology. 2004; 64: 664-8.

7. Ng CF, Chan LW, Wong KT, Cheng CW, Yu SC, Wong WS: Prediction of differential creatinine clearance in chronically obstructed kidneys by non-contrast helical computerized tomography. Int Braz J Urol. 2004; 30: 102-7; discussion 108.

8. El-Ghar ME, Shokeir AA, El-Diasty TA, Refaie HF, Gad HM, El-Dein AB: Contrast enhanced spiral computerized tomography in patients with chronic obstructive uropathy and normal serum creatinine: a single session for anatomical and functional assessment. J Urol. 2004; 172: 985-8.

9. Steckler RE, McLorie GA, Jayanthi VR, Gilday DL, Ash JM, Churchill BM, et al.: Contradictory supranormal differential renal function during nuclear renographic investigation of hydroureteronephrosis. J Urol. 1994; 152: 600-2; discussion 602-3.

Accepted after revision:

October 2, 2008

\section{Correspondence address:}

Dr. Daniel M. Kaplon

Dept. of Surgery/Division of Urology

Rhode Island Hospital

2 Dudley Street, Suite 174

Providence, 02905, RI, USA

Fax: + 1401 444-6947

E-mail:kaplonda@gmail.com

\section{EDITORIAL COMMENT}

Non-invasive estimation of split renal function has been a major challenge for many years, since renal scintigraphy has some limitations (including overestimation of function in obstructive diseases). Helical Computed Tomography (CT), which is considered the most important, self-comprehensive imaging study for renal evaluation, has previously been used to predict split renal function, based on morphological and perfusional criteria (1). However, most of the models require the injection of iodinated 
contrast media, which may be a significant limitation in patients with impaired renal function (given the nephrotoxicity of the contrast agent). Even contrast-enhanced Magnetic Resonance Imaging (MRI), formerly considered a safe method for renal function estimation in patients with impaired renal function, has become a limited exam in this scenario given the apparent association of gadolinium injection and the development of nephrogenic systemic fibrosis in this group of patients.

Summerlin et al. recently published an article showing that split renal function can be correctly calculated using 3D renal volumes obtained on CT images, with good correlation to the results of split post-contrast renal attenuation and renal scintigraphy (2). The article from Dr. Kaplon et al. proposes a practical and straightforward method of calculating split renal function based solely on renal parenchyma thickness, regardless of the use of intravenous contrast media. Given the simple measurements technique, and since it does not involve complicated mathematical models for analysis, it is intuitive to predict that the method could be extrapolated to other imaging techniques, such as ultrasound and non-enhanced MRI, and further investigations will certainly be conducted on these new possibilities.

\section{REFERENCES}

1. Hackstein N, Buch T, Rau WS, Weimer R, Klett R: Split renal function measured by triphasic helical CT. Eur J Radiol. 2007; 61: 303-9.

2. Summerlin AL, Lockhart ME, Strang AM, Kolettis PN, Fineberg NS, Smith JK: Determination of split renal function by 3D reconstruction of $\mathrm{CT}$ angiograms: a comparison with gamma camera renography. AJR Am J Roentgenol. 2008; 191: 1552-8.

Dr. Ronaldo Hueb Baroni Institute of Radiology University of Sao Paulo, USP

Sao Paulo, SP, Brazil E-mail: rbaroni@einstein.br 